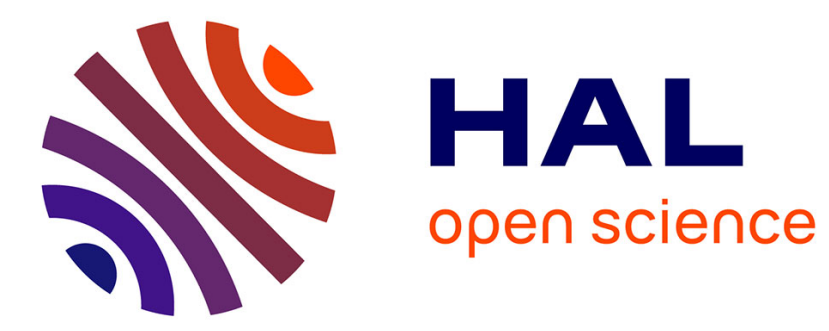

\title{
Clathrin adaptor AP-1 and Stratum act in parallel pathways to control Notch activation in Drosophila Sensory Organ Precursor Cells
}

\author{
Karen Bellec, Mathieu Pinot, Isabelle Gicquel, Roland Le Borgne
}

\section{- To cite this version:}

Karen Bellec, Mathieu Pinot, Isabelle Gicquel, Roland Le Borgne. Clathrin adaptor AP-1 and Stratum act in parallel pathways to control Notch activation in Drosophila Sensory Organ Precursor Cells. 2021. hal-03093034

\section{HAL Id: hal-03093034 https://hal.science/hal-03093034}

Preprint submitted on 3 Jan 2021

HAL is a multi-disciplinary open access archive for the deposit and dissemination of scientific research documents, whether they are published or not. The documents may come from teaching and research institutions in France or abroad, or from public or private research centers.
L'archive ouverte pluridisciplinaire HAL, est destinée au dépôt et à la diffusion de documents scientifiques de niveau recherche, publiés ou non, émanant des établissements d'enseignement et de recherche français ou étrangers, des laboratoires publics ou privés. 


\section{Clathrin adaptor AP-1 and Stratum act in parallel pathways to control Notch activation in Drosophila Sensory Organ Precursor Cells}

Karen Bellec ${ }^{1,2,3}$, Mathieu Pinot ${ }^{1,2}$, Isabelle Gicquel ${ }^{1,2}$, and Roland Le Borgne $e^{1,2, \S}$

${ }^{1}$ Univ Rennes, CNRS, IGDR (Institut de Génétique et Développement de Rennes) - UMR 6290, F- 35000 Rennes, France

${ }^{2}$ Equipe Labellisée Ligue Nationale contre le cancer

${ }^{3}$ Present address: Wolfson Wohl Cancer Research Centre, Institute of Cancer Sciences, University of Glasgow, Glasgow G61 1QH, UK.

${ }^{\S}$ Corresponding author: R.L.B.

Orcid: http://orcid.org/0000-0001-6892-278X

e-mail: roland.leborgne@univ-rennes1.fr

phone: +33223234894

\section{Abstract}


Drosophila sensory organ precursors divide asymmetrically to generate plla/pllb cells whose identity relies on the differential activation of Notch at cytokinesis. While Notch is present apically and basally relative to the midbody at the plla-pllb interface, only the basal pool of Notch is reported to contribute to Notch activation in the plla cell. Correct intra-lineage signalling requires appropriate apico-basal targeting of Notch, its ligand Delta and its trafficking partner Sanpodo. We previously reported that AP-1 and Stratum regulate the intracellular trafficking of Notch and Sanpodo from the trans-Golgi network to the basolateral membrane. Loss of AP-1 or Stratum caused mild Notch gain-of-function phenotypes. Here, we report that the concomitant loss of AP-1 and Stratum results in a much more penetrant Notch gain-of-function phenotype indicating that AP-1 and Strat control two parallel pathways. While unequal partitioning of cell fate determinants and cell polarity were unaffected, Numb-mediated symmetry breaking is impaired. We further observed increased amounts of signaling competent Notch as well as Delta and Sanpodo at the apical plla-pllb interface and the loss of the basal pool of Notch. We propose that AP-1 and Stratum operate in two parallel pathways to ensure the correct apico-basal localization of Notch controlling where receptor activation takes place. 


\section{Introduction}

Cell-cell signaling by the evolutionarily conserved Notch receptor promotes cell fate acquisition in a large variety of developmental processes in metazoans (Artavanis-Tsakonas et al., 1999; Bray, 1998; Kopan and llagan, 2009). In most of cases, Notch receptor is activated by transmembrane ligands present at the plasma membrane of adjacent cells. Following binding to Notch, endocytosis of the ligand induces pulling forces driving a change in the conformation of the Notch extracellular domain thereby unmasking the S2 cleavage site of Notch (Gordon et al., 2015; Langridge and Struhl, 2017; Meloty-Kapella et al., 2012; Seo et al., 2016; Shergill et al., 2012; Wang and Ha, 2013). This regulated cleavage is followed by a constitutive proteolytic cleavage of Notch by the gamma secretase complex (Mumm et al., 2000; Struhl and Adachi, 2000) giving rise to the Notch intracellular domain (NICD), a polypeptide that translocates into the nucleus to act as a transcriptional coactivator (Artavanis-Tsakonas et al., 1999; Bray, 1998; Kopan and Ilagan, 2009). As proteolytic activation of the Notch receptor is irreversible, Notch activation needs to be tightly controlled in time and in space. The model system of asymmetric cell division of the sensory organ precursors (SOPs) in the pupal notum of Drosophila has been instrumental to identify the site of Notch activation at the cell surface. SOPs are polarized epithelial cells that divide asymmetrically within the plane of the epithelium to generate two daughter cells whose fate depends on the differential activation of Notch signaling (Schweisguth, 2015). The differential activation of Notch relies on the unequal partitioning of the two cell fate determinants Neuralized (Neur) and Numb in the anterior SOP daughter cell (Le Borgne and Schweisguth, 2003; Rhyu et al., 1994). Neur promotes the endocytosis of Delta, one of the Notch ligands (Le Borgne and Schweisguth, 2003), while Numb inhibits the recycling of Notch and its cofactor Sanpodo (Spdo) towards the plasma membrane to instead promote their targeting towards late endosomal compartments (Cotton et al., 2013; Couturier et al., 2013; Johnson et al., 2016; Upadhyay et al., 2013). Consequently, the anterior cell adopts the pllb identity while Notch is selectively activated in the posterior cell that adopts the plla fate. Combination of live-imaging, FRAP experiments using NiGFP and photo-tracking of photoconverted NimMaple3 revealed that proteolytic activation of Notch occurs during SOP cytokinesis and that a specific pool of Notch receptors located basal to the midbody is the main contributor to the signaling in the plla cell (Trylinski et al., 2017). These data imply a polarized trafficking of Notch, Delta and Spdo towards this specific subcellular location during cytokinesis.

We previously reported that the clathrin adaptor complex AP-1 regulates the polarized sorting of Notch and Spdo from the trans-Golgi network (TGN) and the recycling endosomes 
(RE) towards the plasma membrane (Benhra et al., 2011). Loss of AP-1 causes stabilization of Notch and Spdo at the adherens junctions following SOP division, a phenotype associated with a mild Notch gain-of-function phenotype (GOF). Unequally partitioned Numb controls the endosomal sorting of Notch/Spdo after asymmetric division and prevents their recycling to the plasma membrane (Couturier et al., 2013). This recycling event relies on AP1 activity (Cotton et al., 2013). We also reported that Stratum (Strat), a chaperone regulating Rab8 recruitment, controls the exit from the Golgi apparatus and the basolateral targeting of Notch, Delta and Spdo (Bellec et al., 2018). As for AP-1, loss of Strat leads to an enrichment in Notch and Spdo at the apical pole of SOP daughter cells associated with a mild Notch GOF phenotype. As AP-1 and Strat/Rab8 both regulate Notch and Spdo trafficking to the basolateral plasma membrane, a possible interpretation of our data is that AP-1 and Strat act in the same transport pathway and therefore are simply fine-tune regulators of NotchDelta trafficking and activation. An alternative explanation could be that AP-1 and Strat function in two parallel pathways (Fig.1A-A') to ensure proper basal localization of the Notch receptor. In this scenario, loss of one of the two components could be at least in part compensated by the other. A prediction of this second hypothesis is that the concomitant loss of AP-1 and Strat would exhibit a stronger phenotype.

In this study, we investigated the consequences of simultaneous disruption of Strat and AP1 function. We report that concomitant impairment of Strat and AP-1 does neither impact the overall apico-basal polarity of epithelial cells, nor the unequal partitioning of Numb and Neur at SOP cytokinesis. However, it does result in increased amounts of Notch, Spdo and Delta at the apical pole of the SOP daughter cells while most of Notch and Spdo, normally localized basally at the plla-pllb interface (Trylinski et al., 2017), appear to be lost. This phenotype is associated with a pllb-to-plla cell fate transformation that is much more penetrant than that of the single $a p-1$ or strat mutants alone. Photoconversion and spatiotemporal monitoring of NimMaple3 in the context of simultaneous impairment of AP-1 and Strat indicate that this fate conversion may be a consequence of aberrant Notch localization. Under simultaneous loss of AP-1 and Strat, Notch receptors localized in excess at the apical pole appear to be able to translocate to nuclei of both SOP daughter cells. This could explain the Notch GOF phenotype as Notch activation may now occur in both SOP daughter cells. We propose a model according which AP-1 and Strat control two parallel transport routes that both contribute to the polarized transport of Notch and Spdo to the basal plla-pllb interface to ensure binary cell fate acquisition at SOP cytokinesis. 


\section{Results}

\section{Simultaneous loss of AP-1 and Strat causes a penetrant Notch GOF phenotype}

To test if AP-1 and Strat function in the same pathway to regulate the activation of Notch signaling pathway, we induced clones of cells homozygote mutant for a null mutation of strat generated by CRISPR (Bellec et al., 2018) in which the $\mu$ subunit of the AP-1 complex (also known as AP-47) was silenced using previously characterized tools (Benhra et al., 2011). Silencing of $A P-47$ is hereafter referred to as loss of AP-1. If Strat and AP-1 act in two different basolateral pathways, we expect the Notch GOF phenotype observed in absence of the two proteins to be much more penetrant. In the wild-type sensory organ (SO), the pllb cell divides two times to generate the internal cells among which there is one Elav positive neuron. The plla cell divides once to generate the external cells among which there is one socket cell identified by Suppressor of Hairless $(\mathrm{Su}(\mathrm{H}))$. Therefore $\mathrm{Su}(\mathrm{H})$ can be used as a read-out of cell fate transformations to monitor the effect of individual or simultaneous impairment of Strat and AP-1 (Fig.1). In agreement with previous studies, mild Notch GOF phenotypes revealed by an excess of $\mathrm{Su}(\mathrm{H})$ positive socket cells were observed in $6,7 \%$ and $7,5 \%$ of SO mutant for strat or depleted of AP-1, respectively (Bellec et al., 2018; Benhra et al., 2011). However simultaneous loss of AP-1 and Strat led to a much stronger Notch GOF phenotype (49\% of transformed SOs, Fig.1B-C). Among them, 34\% of SOs had at least two $\mathrm{Su}(\mathrm{H})$ positive cells with no Elav positive cells indicating that these SOs were already transformed at the two-cell stage (Fig.1C). These results were confirmed using a different method, i.e. silencing both AP1 and Strat using dsRNA (Fig.1C). The strong enhancement of the Notch GOF phenotypes in this lineage analysis indicates that AP-1 and Strat act in two distinct and complementary pathways to regulate Notch activation.

\section{Cell polarity remodeling and unequal partitioning of cell fate determinants upon loss of AP-1 and Strat}

As Strat and AP-1 regulate basolateral trafficking, we first monitored the effect of loss of these two regulators on the distribution of several cell polarity markers. We noticed that, as in AP-1 mutants, the cuticle is thinner and less pigmented than in controls (Fig.S1A-B'). Despite these cuticle defects, the localization of the junctional markers DE-Cadherin, Par3 and Coracle is unaffected by the loss of AP-1 and Strat, suggesting that cell polarity is not altered (Fig.S1C-D). However, time-lapse imaging revealed that Par3, instead of being enriched at the posterior pole of SOP prior to mitosis (Bellaiche et al., 2001; Besson et al., 2015), distributes uniformly at the apical cortex of SOP upon loss of Strat and AP-1 at this stage of the cell cycle (orange arrows, Fig.S1D). This defect in planar cell polarity (PCP) regulation is likely caused by the loss of AP-1 as reported in the Drosophila wing (Carvajal- 
Gonzalez et al., 2012). Despite this defect, during mitosis, Par3 localizes normally at the posterior cortex upon loss of AP-1 and Strat, as it is the case in control SOP (Fig. S2A and S2C). As Par3 is required for the asymmetric localization of Numb and Neur during mitosis (Bellaïche et al., 2001; Langevin et al., 2005), we anticipated that their localization would not be affected in strat mutant SOPs depleted of AP-1. Indeed, we found that Numb and Neur localize asymmetrically during prometaphase in absence of Strat and AP-1 (Fig.2A-B). Liveimaging of Numb::GFP ${ }^{\text {crispr }}$ (Bellec et al., 2018) revealed that Numb is unequally partitioned in the anterior SOP daughter cell, in a similar manner as in the control situation (Fig.2C-D). We conclude that the Notch GOF phenotype is unlikely caused by defective Numb or Neur partitioning during SOP division. These data raise the possibility that the defect originates later, perhaps in the course of cytokinesis.

During cytokinesis, Par3 localizes at the apical cortex and is enriched at the posterior pole of plla cells, as well as at the plla-pllb apical interface (Fig.S2A, t=21 min). In addition, we found that, in the wild-type situation, Par3 localizes in clusters at the lateral plla-pllb interface (Fig.S2A). These Par3 clusters are also positive for Notch bearing a GFP tag in its intracellular domain (Fig.S2A; Houssin and Le Borgne, manuscript in preparation). Strikingly, we found that these lateral Par3/Notch clusters are absent or barely detectable upon loss of Strat and AP-1 activities (Fig.S2C). Instead, upon loss of Strat and AP-1, Par3/Notch clusters now reside within the apical plane of the SOP daughter cells (white arrows, Fig.S2C). Collectively, these data suggest that a defect in the localization of Notch could be causative to the Notch GOF phenotype observed upon loss of Strat and AP-1.

\section{Notch is enriched at the apical plla-pllb interface upon loss of AP-1 and Strat}

To further investigate the effect of AP-1 and Strat on the apico-basal distribution of Notch receptors, we monitored the dynamics of Notch, with a GFP tag in the Notch intracellular domain (Notch::GFP ${ }^{\text {crispr }}$ (Bellec et al, 2018), hereafter called NiGFP) throughout the asymmetric division of the SOP, identified by the nuclear marker Histone2B::RFP expressed under a minimal neur promoter (H2B::RFP, Fig.3). Previously, elegant work from F. Schweisguth laboratory identified two pools of Notch at the plla-pllb interface, apical and basal to the midbody respectively, and provides the compelling evidence that only the subset of receptors located basal to the midbody contributes to signaling (Trylinski et al., 2017). In the control situation, we confirmed the presence of two pools of NiGFP along the apical-basal plla-pllb interface (Fig.3A-A' and S2A). NiGFP is transiently detected at the apical plla-pllb interface at 6-9 min after anaphase onset with a signal intensity peaking at 
15-20 min prior to progressively disappear at $30 \mathrm{~min}$ (Fig.3A-B, S2A-B and Movie S1). Basal to the midbody, NiGFP localizes in lateral clusters, which appear 6-9 min following the anaphase onset and persist longer than the apical pool of NiGFP (up to 45 min after the anaphase onset; Fig.3A-B' and Fig.S2A). While NiGFP colocalizes with Par3 in these lateral clusters in wild-type (Fig.3A-A', 3B' and S2A), upon loss of Strat and AP-1, the NiGFP-Par3 lateral clusters were barely detectable (Fig.3B'-C' and S2C). Instead, we found that NiGFP appears in higher amounts at the apical plla-pllb interface cells 6-9 minutes following the anaphase onset and persists there for longer periods of time compared to the control (at least 78 min post-anaphase versus 30 min in the control; Fig.3B-C', S2C and Movie S2). In addition to accumulating at the apical interface, NiGFP also localizes in intracellular compartments in the apical plane, some of which are positive for Par3 (white arrows, Fig.3C-C', S2C and Movie S2). Since lateral clusters from which Notch is activated (Trylinski et al., 2017) are barely detectable upon loss of Strat and AP-1, our results prompt the hypothesis that the Notch GOF phenotype originates from the ectopic apically enriched pool of NiGFP.

Spdo, Neur and Delta are distributed with Notch at the apical plla-pllb interface upon loss of Strat and AP-1

Previously, Notch phenotypes observed in AP-1 or strat mutant were associated with defects in Spdo and Delta localization. This prompted us to study the localization of Spdo and Delta on fixed specimens. As previously described, in the control situation, Spdo is faintly detected at the apical pole of SOP daughter cells and localizes predominantly in endosomes in the pllb cell. In the plla cell, Spdo distributes not only in endosomes (yellow arrows, Fig.4A) but also at the basolateral plasma membrane (red arrows, Fig.4A; (Cotton et al., 2013; Couturier et al., 2013; Hutterer and Knoblich, 2005; Langevin et al., 2005). In contrast upon loss of Strat and AP-1, Spdo is still detected in dotted intracellular structures in SOP daughter cells but is no longer detected at the basolateral plla-pllb interface (Fig.4A). Instead, Spdo is enriched at the apical plasma membrane as well as at the apical plla-pllb interface ( $n=12 / 14$, white arrows, Fig.4A-B).

Notch activation requires Neur-mediated Delta endocytosis. In the wild-type situation, Delta and Neur localize at the lateral plla-pllb interface (Trylinski et al, 2017; Fig.4C-E). While Neur is uniformly distributed at the apical pole in cytokinesis (Trylinski et al, 2017; Fig.4E), Delta is barely detected at the apical plla-pllb interface (Fig.4C-D). The loss of Strat and AP1 does not appear to significantly alter the location of the Neur at the plla-pllb interface 
(Fig.4E) but it results in an increase in the number of SOPs with Delta labelling at the apical plla-pllb interface $(60 \%$ of SOs against $44 \%$ in the control situation, Fig.4C-D). Therefore, loss of Strat and AP-1 causes abnormally high levels of Notch/Delta and Spdo at the apical plla-pllb interface at the expense of their localization to the basolateral interface. These observations raised the question of whether Delta-mediated Notch activation can take place at the apical interface upon loss of Strat and AP-1. We tested the dynamics of the ectopic apical Notch pool using photobleaching. The apical pool of NiGFP in absence of Strat and AP-1 appears to be constantly replenished and rapidly recovered (yellow arrows, Fig.S3A). We noticed that 120 seconds after photobleaching, the fluorescence recovery reaches its maximum in control and upon loss of Strat and AP-1. However, the mobile fraction is around $67 \%+/-30 \%$ upon loss of Strat and AP-1 compared to $31 \%+/-15 \%$ in a control situation (Fig.S3B) with a t1/2 of around 40 seconds $+/-7$ seconds and a t1/2 of 37 seconds $+/-11$ seconds, respectively (Fig.SC). The high recovery rate of the apical pool of NiGFP raises the question of its destiny and is in agreement with Delta-mediated Notch activation that can take place at the apical interface upon loss of Strat and AP-1.

\section{The apical pool of Notch contributes to the Notch GOF phenotype caused by the loss of Strat and AP-1}

To further investigate the ability of the apical pool of Notch to be cleaved and transferred into the nucleus, we photoconverted Notch receptors present at the membrane and measured the presence of photoconverted Notch in the nuclei of daughter cells using NimMaple3 (Trylinski et al., 2017). We first validated our ability to photoconvert nuclear NimMaple3 in the wild-type SOP daughter cells and in SOP daughter cells deprived of Strat and AP-1, 30 minutes after the anaphase onset (Fig.5A-B). The plasma membrane of SOPs and daughter cells were identified using GAP43::IRFP670 (thereafter referred to as GAP43::IR) expressed under a minimal neur promoter (Fig.5A'). As reported, in a control situation, higher levels of photoconverted nuclear Notch were found in the plla cell compared to the pllb cell, thus reflecting the differential activation of the Notch signaling (Trylinski et al., 2017, Fig.5B'). On the contrary, in absence of Strat and AP-1, the amount of photoconverted nuclear Notch is similar between the two daughter cells, in agreement with the Notch GOF phenotype described (Fig.5B'). Once photoconverted, the nuclear signal rapidly decreased over time $(\mathrm{t} 1 / 2=5 \mathrm{~min})$, in agreement with the known instability of nuclear NICD (Fig.S4D-D', (GuptaRossi et al., 2001; Jarriault et al., 1995)).

We then tested the precision of NimMaple3 photoconversion in controls. Photoconversion at the level of the apical plla-pllb interface remained restricted to the apical plane as no 
undesired photoconversion occurred deeper in the cell (Fig.S4A-A'). In contrast, photoconversion within a $\mathrm{ROI}$ encompassing the lateral plla-pllb plasma membrane interface lead to unwanted photoconversion in the adjacent nuclei (Fig.S4B-B') and at the apical level over a diameter of about 3-4 cells (Fig.S4C-C'). Therefore, the inability to ensure precise photoconversion on the lateral interface prevents testing by photoconversion whether Notch is activated from the apical, the lateral or from both pools of receptors in controls. This technical caveat is, however, not a concern upon loss of Strat and AP-1 as NimMaple3 signal (or NiGFP) is barely detected at the lateral interface (Fig.3B'-C' and Fig.S2C). When we photoconverted Notch at apical plla-pllb interface in this context, we found that Notch can enter in both nuclei that result from the division (Fig.5C-C"). The levels of photoconverted Notch measured in both nuclei (Fig.5C"', cell $i$ highlighted in yellow on panel $\mathbf{5} \mathbf{C}^{\prime}$ ) are further higher than those found in the nuclei of a neighbouring SOP that divides in the same time, but that was not exposed to photoconversion (Fig.5C'”, cell ii highlighted in blue on panel 5C'). On average, the contribution of the apical pool of Notch when Strat and AP-1 are simultaneously impaired seems identical between the two nuclei as demonstrated by the ratio of photoconverted nuclear signal plla/plla-like close to 1 and the absence of significant differences in the amount of photoconverted nuclear signal between the two cells (Fig.5D-D'). Taken together, these data demonstrate that, in absence of Strat and AP-1, the apical enrichment of Notch at the interface is source of activated receptors present in nuclei of both daughter cells therefore causing Notch GOF phenotype.

\section{Discussion}

\section{Strat and AP-1 act redundantly in the basolateral transport of Notch signaling components in SOPs}

Previous work showed that Strat/Rab8 control the transport of Notch, Delta and Spdo from the TGN to the basolateral pole (Bellec et al., 2018) and that AP-1 controls the targeting of Notch and Spdo from the TGN to the basolateral membrane via the recycling endosomes (Benhra et al., 2011; Cotton et al., 2013). Here, we report that upon concomitant loss of Strat and AP-1, Notch, Delta and Spdo are no longer detected in clusters at the lateral SOP daughter cells interface and are instead enriched at the apical interface (Fig.6; Bellec et al., 2018; Benhra et al., 2011; Cotton et al., 2013). We found that AP-1 and Strat act redundantly to control the basolateral targeting of Notch, Delta and Spdo in SOP daughter cells explaining the partial compensation of one by the other. These results are in agreement with previous studies demonstrating the involvement of Rab8, whose activity and localization is controlled by Strat in Drosophila (Bellec et al., 2018; Devergne et al., 2017), in the basolateral sorting of proteins (Ang et al., 2003; Henry and Sheff, 2008; Huber et al., 1993). 
However the role of Rab8is not restricted to the basolateral transport as the loss of one or the other has also been previously associated to defects in apical targeting and secretion (Castillon et al., 2018; Gillard et al., 2015; Holloway et al., 2013; Nakajo et al., 2016; Norgate et al., 2006; Sato et al., 2014; Sato et al., 2007; Zhang et al., 2012). In the same way, the role of AP-1 is not restricted to the basolateral transport. In our study, we described a thinner and less pigmented cuticle in absence of Strat and AP-1. These phenotypes could be caused by a defective AP-1 dependent transport of the Menkes Copper transporter ATP7a that regulates cuticle pigmentation (Holloway et al., 2013; Norgate et al., 2006) and/or by a reduction in apical secretion of cuticle components that may rely on AP-1 function as does the apical glue granule secretion in salivary glands (Burgess et al., 2011). These apparent contradictory results might be explained by a distinct Rab8- or AP-1- mediated sorting depending on the cargo to be transported or on the tissue. In addition, sorting might occur at the level of RE. If a protein that does not normally transit via $R E$ is found to be present in $R E$ in a mutant background, it may be sorted and transported to an unusual apical location by default.

\section{Notch signaling activation can take place at the apical interface of the SOP daughter cells}

How can Notch be activated in the absence of lateral clusters, the main contributors of intralineage Notch activation (Trylinski et al., 2017), in absence of Strat and AP-1? Our work indicates that Notch activation can take place at the apical plla-pllb interface. Indeed, phototracking of photoconverted NimMaple3 in the strat mutant background deprived of AP-1 revealed that the apical pool of Notch is an active pool of Notch in terms of signaling, contributing to the nuclear NICD signal in both daughter cells. However, we cannot exclude the possibility that a few, hard to detect, lateral clusters could also contribute to Notch activation upon loss of AP-1 and Strat. In the same way, we cannot exclude that Notch activation can take place from the Notch positive compartments observed at the apical pole of SOP daughter cells upon loss of Strat and AP-1.

The fact that Sec15, a subunit of the exocyst complex, and Arp2/3-WASp (Actin-related protein 2/3-Wiskott-Aldrich syndrome protein) are involved in apical trafficking of Spdo and Delta, respectively, and are required for Notch-Delta signaling is consistent with our proposal of apical activation of Notch (Jafar-Nejad et al., 2005; Rajan et al., 2009). However, the weak contribution of the apical pool of receptors to the Notch activation described in the control situation (Trylinski et al., 2017) suggests that apical activation can only occur when a given threshold of Notch, Delta and Spdo is reached, such as in strat SOs depleted of AP-1. In line with previous reports (Trylinski et al., 2017), our study further illustrates how the 
apico-basal targeting of Notch, Spdo and Delta along the plla-pllb interface limits signaling between SOP daughter cells at the exit of mitosis. The question of whether this mechanism is conserved awaits further investigation, but given the key role played by Notch in the acquisition of cell fate in vertebrates, it is conceivable that the regulation described in this study may be general.

\section{Loss of Strat and AP-1 causes Notch activation in both SOP daughter cells}

As reported in this study, the loss of Strat and AP-1 leads to a Notch GOF despite the unequal partitioning of Numb. This phenotype can be explained by the lack of AP-1 that prevents Numb to repress the Notch-Spdo recycling normally driving endosomal degradation of Notch-Spdo (Cotton et al., 2013; Couturier et al., 2013; Johnson et al., 2016). Thus, Numb is not able to repress Notch activation upon loss of AP-1 and Strat, thereby unable to trigger symmetry breaking in Notch signaling at SOP cytokinesis.

Proteolytic activation of Notch requires the binding of the ligand and its subsequent Neurmediated endocytosis. While Neur is unequally localized during SOP division (Le Borgne and Schweisguth, 2003), Neur::GFP was shown to localize in both SOP daughter cell apical cortex and to be enriched at the apical plla-pllb interface following SOP division (Trylinski et al., 2017). As Delta and Neur localizes at the apical SOP daughter cell interface upon loss of Strat and AP-1 (this work), we propose that Neur can promote endocytosis of Delta in both daughter cells from the apical interface, thus leading to the bidirectional Notch proteolytic activation. In agreement with this proposal according which Neur might be active in the two SOP daughter cells, it is interesting to note that in numb mutant cells, despite the unequal inheritance of Neur, Delta-dependent Notch activation takes place in both daughter cells, as observed here upon loss of Strat and AP-1.

\section{Acknowledgements}

We thank the Bloomington Stock Center, the Vienna Drosophila RNAi Center and the National Institute of Genetics Fly Stock Center for providing fly stocks. We also thank S. Dutertre and X. Pinson from the Microscopy Rennes Imaging Center-BIOSIT (France). The monoclonal antibodies against Elav, Cut, Cora and DE-Cad were obtained from the Developmental Studies Hybridoma Bank, generated under the auspices of the National Institute of Child Health and Human Development, and maintained by the University of lowa Department of Biological Sciences. We thank Dr. J. Januschke, University of Dundee for sharing the Par3::Scarlet Drosophila line prior to publication. We thank Dr. J. Januschke and 
the members of R.L.B.'s laboratory for helpful discussions and critical reading of the manuscript.

\section{Competing interests}

The authors declare no competing or financial interests.

\section{Author contributions}

Conceptualization: K.B., R.L.B.; Methodology: K.B., M.P., I.G., R.L.B.; Formal analysis: K.B., M.P., R.L.B.; Investigation: K.B., M.P., R.L.B.; Resources: K.B., I.G., R.L.B.; Writing original draft: K.B.; Writing - review \& editing: K.B., M.P., R.L.B.; Visualization: K.B., M.P., R.L.B.; Supervision: R.L.B.; Project administration: R.L.B.; Funding acquisition: K.B., R.L.B.

\section{Funding}

This work was supported in part by the ARED programme from the Région Bretagne/Agence Nationale de la Recherche (ANR-16-CE13-004-01), the Fondation pour la Recherche Médicale (FDT20170436864 to K.B.), La Ligue contre le Cancer-Equipe Labellisée (R.L.B.) and the Association Nationale de la Recherche et de la Technologie programme PRC Vie, santé et bien-être CytoSIGN (ANR-16-CE13-004-01 to R.L.B.). 


\section{Materials and Methods}

\section{Drosophila stocks and genetics}

Drosophila melanogaster stocks were maintained and crossed at $25^{\circ} \mathrm{C}$. Mitotic clones were induced using the FLP-FRT technique using the $h s$-FLP and by heat shocking $(2 \times 60 \mathrm{~min}$ at $37^{\circ} \mathrm{C}$ ) at second and early third instar larvae. pnr-GAL4 was used to drive the expression of the $A P-47^{d s R N A}$ and the strat ${ }^{d s R N A}$. The following stocks were used in this study:

Figure 1B: $y$, $h s$-FLP $(/ w)$; Ubi-GFP $n / s$, FRT40A/Ubi-GFP $n / s$, FRT40A and $y, w$, hs-FLP (/w); Ubi-GFP n/s, FRT40A/strat, FRT40A; pnr-GAL4/AP-47dsRNA (stock \#24017 from Vienna Drosophila Resource Center, $w^{1118}$; P(GD14206)v24017/TM3)

Figure 1C: $y$, hs-FLP (/w); Ubi-GFP nls, FRT40A/Ubi-GFP nls, FRT40A and $y$, hs-FLP (/w); Ubi-GFP nls, FRT40A/strat, FRT40A and $y$, w, hs-FLP (/w); Ubi-GFP nls, FRT40A/strat, FRT40A; pnr-GAL4/AP-4 $7^{d s R N A}$ and $y, w$, NimMaple3/Y; strat dsRNA $/+;$ pnr-GAL4, neurGAP43::IRFP670/AP-47 $7^{d s R N A}$

Figure 2A: Cad::GFP/+; pnr-GAL4/+; +/CyO::CFP; pnr-GAL4/+ and $y, w$, hs-FLP (/w); UbiGFP n/s, FRT40A/strat, FRT40A; pnr-GAL4/AP-47dsRNA

Figure 2B: $y, w$, hs-FLP/ NiDendra, neur-H2B::RFP; Ubi-GFP n/s, FRT40A/FRT40A; pnrGAL4/+ and $y, w$, hs-FLP/NiDendra, neur-H2B::RFP; Ubi-GFP n/s, FRT40A/strat, FRT40A; pnr-GAL4/AP-4 $7^{d s R N A}$

Figure 2C: $y, h s-F L P(w)$; Ubi-RFP nls, FRT40A/Numb::GFP ${ }^{\text {crispr }}$, FRT40A

Figure 2D: $w$, hs-FLP neur-H2B::RFP/w; Ubi-GFP nls, FRT40A strat, Numb::GFP ${ }^{\text {crispr }}$, FRT40A; pnr-GAL4/ AP-4 $7^{d s R N A}$

Figure 3: $y$, w, hs-FLP/NiGFP, neur-H2B::RFP; Ubi-GFP nls, FRT40A/FRT40A; pnr-GAL4/+ and $y, w$, hs-FLP/NiGFP, neur-H2B::RFP; Ubi-GFP n/s, FRT40A/strat, FRT40A; pnr-GAL4/ $A P-47^{d s R N A}$ and $y, w$, NiGFP, Par3::Scarlet; +/+; pnr-GAL4/+ and $y, w$, NiGFP,

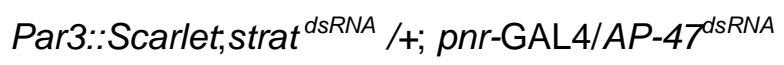

Figure 4A-B: $W 1118$ and $y, w$, hs-FLP $(/ w)$; Ubi-GFP nls, FRT40A/strat, FRT40A; pnrGAL4/AP-47dsRNA

Figure 4C-D: $y$, w, hs-FLP/ NiDendra, neur-H2B::RFP; Ubi-GFP n/s, FRT40A/FRT40A; pnrGAL4/+ and $y, w$, hs-FLP/NiDendra, neur-H2B::RFP; Ubi-GFP n/s, FRT40A/strat, FRT40A; pnr-GAL4/AP-4 $7^{d s R N A}$ 
Figure 4E: $y, w$, hs-FLP/NiDendra, neur-H2B::RFP; Ubi-GFP nls, FRT40A/FRT40A; pnrGAL4/+ and $y, w$, hs-FLP/NiDendra, neur-H2B::RFP; Ubi-GFP n/s, FRT40A/strat, FRT40A; pnr-GAL4/AP-4 $7^{d s R N A}$ and $y, w, N i G F P$, Par3::Scarlet; strat $t^{d s R N A} /+;$ pnr-GAL4/AP-4 $7^{d s R N A}$

Figure 5: $y, w$, NimMaple3/Y; strat ${ }^{d s R N A} /+;$ pnr-GAL4, neur-GAP43::IRFP670/AP-47 ${ }^{d s R N A}$

Figure S1A-A': NiDendra, neur-H2B::RFP; +/+

Figure S1B-B': $y, w$, hs-FLP/NiDendra, neur-H2B::RFP; Ubi-GFP nls, FRT40A/strat, FRT40A; pnr-GAL4/AP-4 $7^{d s R N A}$

Figure S1C: $y, w, h s-F L P(/ w)$; Ubi-GFP nls, FRT40A/strat, FRT40A; pnr-GAL4/AP-47dsRNA

Figure S1D: $y, w$, NiGFP, Par3::Scarlet; +/+; pnr-GAL4/+ and $y, w$, NiGFP, Par3::Scarlet; strat $t^{d s R N A} /+;$ pnr-GAL4/AP-4 $7^{d s R N A}$

Figure S2: $y, w$, NiGFP, Par3::Scarlet; +/+; pnr-GAL4/+ and $y, w$, NiGFP, Par3::Scarlet;strat dsRNA $/+;$ pnr-GAL4/AP-47dsRNA

Figure S3A: $y, w, N i G F P$, Par3::Scarlet; strat $t^{d s R A} /+;$ pnr-GAL4/AP-47dsRNA

Figure S3B-C: $y, w$, NiGFP5, neur-H2B::RFP (control) and $y, w, N i G F P$, Par3::Scarlet; strat $t^{d s R N A} /+;$ pnr-GAL4/AP-4 $7^{\text {dsRNA }}$

Figure S4A-C: $y, w$, NimMaple3/Y; + /+ pnr-GAL4, neur-GAP43::IRFP670/+

Figure S4D-D': $y, w$, NimMaple3/Y; strat ${ }^{d s R N A} /+;$ pnr-GAL4, neur-GAP43::IRFP670/AP-47 dsRNA

Movie S1: $y, w$, hs-FLP/ NiGFP, neur-H2B::RFP; Ubi-GFP nls, FRT40A/FRT40A; pnrGAL4/+

Movie S2: $y, w$, hs-FLP/NiGFP, neur-H2B::RFP; Ubi-GFP nls, FRT40A/strat, FRT40A; pnrGAL4/ $A P-47^{d s R N A}$

\section{Immunofluorescence and antibodies}

Pupae were aged for 16.5 to $18.5 \mathrm{~h}$ after puparium formation (APF) for SOPs and SOPs daughter cells analysis and were aged for $24 \mathrm{~h}$ to $28 \mathrm{~h}$ APF for lineage analysis. Pupae were dissected in $1 \mathrm{x}$ phosphate-buffered saline (1x PBS) and then fixed for $15 \mathrm{~min}$ in $4 \%$ paraformaldehyde at room temperature. Dissection and staining conditions were essentially as previously described (Le Borgne and Schweisguth, 2003). Primary antibodies used were rat anti-Elav (7E10, Developmental Studies Hybridoma Bank (DSHB), 1:200), goat anti- 
$\mathrm{Su}(\mathrm{H})$ (sc15813, Santa Cruz, 1:500), mouse anti-Cut (2B10, DSHB, 1:500), rabbit anti-Neur ((Lai et al., 2001), 1:1000), goat anti-Numb (SC23579, Santa Cruz, 1:200), rabbit anti-Spdo (a kind gift from J. Skeath, 1:2000) (O'Connor-Giles and Skeath, 2003), mouse anti-DECD (C594.9B, DSHB, 1:200), mouse anti-Cora (C615.16, DSHB, 1:500), rat anti-DE-Cad (DCAD2, DSHB, 1:500) and rabbit anti-Dendra (Antibodies-online.com, ABIN361314, 1:1000). Cy2-, Cy3- and Cy5-coupled secondary antibodies (1:400) were from Jackson's Laboratories.

\section{Generation of NiDendra}

The NiDendra construct was generated using the CRISPR/Cas9 method as previously described (Gratz et al., 2013a; Gratz et al., 2013b). As for the NiGFP construct, the following gRNAs were used: 5'-AACTTGAA|TGGATTGAACCC GGG-3' and 5'CGAACTGG|AGGGTTCTCCTGTTG-3' to introduce Cas9 cuts in exon 6 (Bellec et al., 2018). The Dendra and the 3xP3-DsRed cassette flanked by GVG linkers and by loxP, respectively, were introduced at the previously described position (NiYFP4) (Couturier et al., 2012). Homology arms 1 and 2 were of $1064 \mathrm{bp}$ and $1263 \mathrm{bp}$ in length, respectively. Injection was performed by Bestgene in the $y w$, attP40(nos-cas9)/Cyo stock. The correct position of the Dendra and the DsRed cassette was verified by PCR and sequencing. The DsRed was then removed by crossings with if/Cyo, Cre, w stock. The insertion of Dendra tag did not alter the functionality of Notch. Anti-Dendra signal is detected in the plla nucleus indicating that processed NiDendra is translocated into the nucleus (data not shown).

\section{Imaging}

Images of fixed nota were acquired with a Leica SPE confocal microscope and a Zeiss Airyscan microscope (LSM 880 with AiryScan module). Live imaging of NiGFP with Par3::Scarlet was performed with a Leica SPE confocal microscope. Live imaging of NiGFP in wild-type SOP and in strat SOP expressing pnr-GAL4>AP-47dsRNA (Fig.S2) was performed with a Zeiss Airyscan microscope (LSM 880 with AiryScan module). All images were processed and assembled using ImageJ 1.48 and Adobe Illustrator.

\section{Quantification of the enrichment of Notch at the apical and basal interface}

To quantify the signal of NiGFP at the apical interface, we measured the signal in a manually drawn area on sum slices of the two apical planes where the Notch signal at the apical interface is the strongest. We also measured the signal between two epithelial cells within an equivalent drawn area and on the same sum slices. Then, we calculated the following ratio: Average fluorescence intensity at the apical interface between SOP daughter cells/Average 
fluorescence intensity at the apical interface between epithelial cells. This ratio has been calculated for each time point. In absence of AP-1 and Strat, the manually drawn area was minimized to avoid considering the apical punctate structures positive for Notch.

To quantify the signal of NiGFP at the basal interface, we measured the signal in a manually drawn area between nuclei on sum slices of two planes. In a wild-type situation, we did the sum slices on planes where we observed basolateral clusters positive for Par3 and Notch. In absence of AP-1 and Strat and in absence of clusters, we did the sum slices on equivalent planes located between the two nuclei. In parallel, we measured the background fluorescence noise on the same sum slices, with the same manually drawn area, in the cytoplasm of epidermal cells. Then, we calculated the following ratio: Average fluorescence intensity at the basal interface between SOP daughter cells - the background fluorescence noise /Average fluorescence intensity at the apical interface between epithelial cells. This ratio has been calculated for each time point.

\section{Quantification of the apical enrichment of Spdon and Delta}

The fluorescence intensity was calculated with ImageJ, on two-cell stages, as previously described (Bellec et al., 2018). For Spdo, the average fluorescence intensity was measured in a manually drawn area on sum slices of the two most apical planes, where Spdo is enriched. The background noise was measured in the same way and subtracted from the apical intensity value. For the Delta, the same protocol was applied: the average fluorescence intensity was measured in a manually drawn area on sum slices of the two apical planes where Delta is enriched at the interface between the two daughter cells. These values were divided by the average intensity measured at the apical interface between epidermal cells, on the same sum slices and with the same manually drawn area. The background noise was measured in the same way and subtracted from the apical intensity values.

\section{FRAP experiments}

Photobleaching of NiGFP/NiGFP5 was performed using a Zeiss AiryScan (LSM 880 with AiryScan module) with the $488 \mathrm{~nm}$ laser wavelength $(20 \mathrm{~mW})$ at $90 \%$ of the maximal power and by using the $63 x$ oil objective (NA 1.4). Two consecutive iterations were performed. The FRAP area was defined by the plla/pllb interface. A control area corresponding to an adjacent epidermal cell interface was measured to obtain the general photobleaching of the sample over the period of acquisition. All FRAP data were analyzed using the easyFRAP software tool (https://easyfrap.vmnet.upatras.gr/?AspxAutoDetectCookieSupport=1). Half- 
time $t 1 / 2$ and mobile fraction were then extracted with GraphPAD Prism software using a one-component equation.

\section{Photoconversion experiments}

Photoconversion of NimMaple3 was performed using a Zeiss AiryScan (LSM 880 with AiryScan module) with a $405 \mathrm{~nm}$ diode $(30 \mathrm{~mW})$ at $1.8 \%$ power (40 iterations and pixel dwell time: $1.52 \mu \mathrm{s})$ according to Trylinski et al., 2017.

In nuclear photoconversion assays (Fig.5A-B), the photoconverted ROI (dashed yellow rectangles) were defined using the GAP43::IR expressed under the neur promoter. Because GAP43::IR is excluded from the nuclei, nuclei were defined using an area within cells where the GAP43::IR signal background is the lowest. Nuclei measurement (dashed white circles, Fig.5B and Fig.5C'”) were then performed using a circular ROI corresponding to the half of the presumptive diameter of nuclei with the center of the ROI positioned on the center of mass of nuclei.

Apical photoconversions in pnr-GAL4>strat ${ }^{d s R N A}, A P-47^{d s R N A}$ SOP daughter cells were performed at 15, 20, 25 and 30 minutes after anaphase transition Before photoconversion, z-stacks were performed to localize the apical interface using GAP43::IR only first, and then with green NimMaple3. A z-stack was then acquired 35 minutes after the anaphase onset to quantify nuclear NimMaple3. No threshold has been applied.

\section{Statistical analysis}

Statistical analyses were carried out using the GraphPad Prism 6.07 software. A two-way ANOVA was performed for the quantification of the enrichment of Notch at the apical and at the basal plla-pllb interface with a multiple comparison Bonferroni test. For other quantifications, we performed a t test if the data followed a normal distribution or a Wilcoxon test or a Mann-Whitney test if the data did not follow a normal distribution. The normal distribution was tested by a Shapiro test. Statistical significances are represented as follows: not significant (ns) $\geq 0.05 ;{ }^{\star} P<0.05 ;{ }^{* *} P<0.01 ;{ }^{* \star *} P<0.001$ and ${ }^{* \star * \star} P<0.0001$. 


\section{Figure Legends}

Figure 1: Loss of Strat and AP-1 causes Notch gain-of-function phenotype within SO lineage

A-A'. Schematic representations of the involvement of AP-1 and Strat in the same $(\mathbf{A})$ or in distinct (A') basolateral transport pathway. B. Projection of confocal sections of a pupal notum at $24 \mathrm{~h}$ APF in a wild-type organ or in strat SOP expressing pnr-GAL4>AP-47dsRNA. strat mutant cells were identified by the absence of the nuclear marker nls-GFP (grey). Sockets were identified with $\mathrm{Su}(\mathrm{H})(\operatorname{anti}-\mathrm{Su}(\mathrm{H})$, green) and neurons with Elav (anti-Elav, red). In the wild-type organ, cells composing the SO are identified with Cut (anti-Cut, blue). Scale bar is $5 \mu \mathrm{m}$. Yellow and red arrows show transformed SOs containing 3 to $4 \mathrm{Su}(\mathrm{H})$ positive cells or $2 \mathrm{Su}(\mathrm{H})$ positive cells without neuron, respectively. C. Percentage of transformed organs at 24h APF in wild-type $(\mathrm{n}=204)$, in strat, pnr-GAL4> $+(\mathrm{n}=328)$, in pnrGAL4 $>A P-47^{d s R N A} \quad(\mathrm{n}=362)$, in strat, pnr-GAL4>AP-4 $7^{d s R N A} \quad(\mathrm{n}=254)$ and in pnrGAL4>strat ${ }^{d s R N A}, A P-47^{d s R N A}(\mathrm{n}=144)$.

\section{Figure 2: Loss of Strat and AP-1 does not affect the localization of Numb}

A. Localization of Numb (anti-Numb, green) in wild-type ( $n=22$ of prophase/prometaphase and dividing SOPs) and in strat SOP expressing pnr-GAL4>AP-4 $7^{d s R N A}$ ( $\mathrm{n}=16$ of prophase/prometaphase and dividing SOPs). SOPs and SOPs daughter cells were identified with Cut (anti-Cut, red). B. Localization of Neur (anti-Neur, green) in wild-type ( $n=14$ of prophase/prometaphase and dividing SOPs) and in strat SOP expressing pnr-GAL4>AP$47^{d s R N A}$ ( $n=7$ of prophase/prometaphase and dividing SOPs). SOPs and SOPs daughter cells were identified with Histone2B::RFP expressed under the neur promoter (red). Similar phenotype was observed in the pnr-GAL4>strat ${ }^{d s R N A}, A P-47^{d s R N A}$ (data not shown, $\mathrm{n}=8$ of prophase/prometaphase and dividing SOPs). C-D. Time-lapse imaging of Numb::GFP ${ }^{\text {crispr }}$ (green) in dividing wild-type SOP expressing Histone2B::RFP under the neur promoter (red; C, $\mathrm{n}=10$ ) and in dividing strat SOP expressing Histone2B::RFP under the neur promoter (red) and pnr-GAL4>AP-4 $7^{d s R N A}(\mathbf{D}, \mathrm{n}=15)$. Time is minute:second and the time 00:00 corresponds to the SOP anaphase onset. Scale bar is $5 \mu \mathrm{m}$.

Figure 3: Notch is enriched at the apical plla-pllb interface in the absence of Strat and AP-1

A. Time-lapse imaging of NiGFP (green) and Histone2B::RFP expressed under the neur promoter (red) in dividing wild-type SOP $(n=10)$. A'. Schematic representation of Notch localization at $t=9 \mathrm{~min}$ and $t=33 \mathrm{~min}$ in wild-type SOP daughter cells. B. Quantification of the 
fluorescence intensity of NiGFP at the apical plla-pllb interface of wild-type SOP daughter cells (white) and strat SOP daughter cells expressing pnr-GAL4>AP-4 $7^{d s R N A}$ (red). Boxes extend from the $25^{\text {th }}$ to $75^{\text {th }}$ percentiles and the line in boxes represents the median. The whiskers go down to the smallest value and up to the largest. The statistics were carried out from $\mathrm{t}=0$ to $30 \mathrm{~min}$ and $\mathrm{n}=10$ for both conditions (ns $\geq 0.05$; ${ }^{*} P<0.05$ and ${ }^{* * * *} P<0.0001$ ). Statistics were carried out until 30min as most of wild-type SOPs movies stops at 30min. B'. Quantification of the fluorescence intensity of NiGFP at the basal plla-pllb interface of wildtype SOP daughter cells (white, $\mathrm{n}=11$ ) and SOP daughter cells expressing pnrGAL4>strat ${ }^{d s R N A}, A P-47^{d s R N A}$ (red, $\mathrm{n}=22$ ). Boxes extend from the $25^{\text {th }}$ to $75^{\text {th }}$ percentiles and the line in boxes represents the median. The whiskers go down to the smallest value and up to the largest ( $\mathrm{ns} \geq 0.05$ ). C. Time-lapse imaging of NiGFP (green) and Histone2B::RFP expressed under the neur promoter (red) in dividing strat SOP expressing pnr-GAL4>AP$47^{d s R N A}(\mathrm{n}=10 / 12)$. C'. Schematic representation of Notch localization at $\mathrm{t}=9 \mathrm{~min}$ and $\mathrm{t}=39 \mathrm{~min}$ in strat SOP daughter cells expressing pnr-GAL4>AP-4 $7^{d s R N A}$. Dashed white lines highlight SOP and SOP daughter cells. Yellow arrows point to the enrichment of NiGFP at the apical interface between SOP daughter cells and white arrows point to apical compartments positive for NiGFP. Dashed yellow rectangles highlight compartments positive for NiGFP at the basolateral interface between wild-type SOP daughter cells. Basal views are a maximum projection of three confocal slices (Sum projection). Time is in hour:minute and the time 00:00 corresponds to the SOP anaphase onset. Scale bar is $5 \mu \mathrm{m}$.

Figure 4: Spdo, Neur and Delta are enriched at the apical plla-pllb interface in absence of Strat and AP-1

A. Localization of Spdo (anti-Spdo) in wild-type SOP daughter cells and in strat SOP daughter cells expressing pnr-GAL4>AP-4 $7^{d s R N A}$. White arrows point to the enrichment of Spdo at the apical interface between SOP daughter cells, red arrows point the enrichment of Spdo at the basolateral plasma membrane and yellow arrows point to endosomes positive for Spdo. B. Quantification of the fluorescence intensity of Spdo at the apical pole of wild-

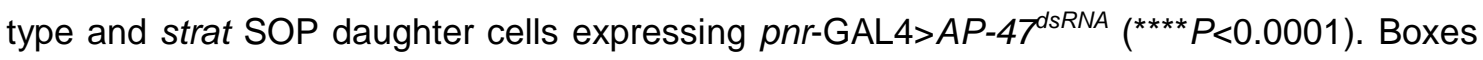
extend from the $25^{\text {th }}$ to $75^{\text {th }}$ percentiles and the line in boxes represents the median. The squares and the triangle show maximum values. C. Localization of NiDendra (anti-Dendra, green), Histone2B::RFP expressed under the neur promoter (blue) and Delta (anti-Delta, red) in wild-type SOP daughter cells and in strat SOP daughter cells expressing pnrGAL4 $>A P-47^{d s R N A}$. White arrows point to the enrichment of Notch and Delta at the apical interface between SOP daughter cells. A projection (Maximum intensity) of the three most apical planes is shown for the apical view. D. Quantification of the fluorescence intensity of Delta at the apical interface of wild-type and strat SOP daughter cells expressing pnr- 
GAL4>AP-4 $7^{d s R N A}\left({ }^{* \star *} P<0.001\right)$. Boxes extend from the $25^{\text {th }}$ to $75^{\text {th }}$ percentiles and the line in boxes represents the median. The triangle shows the maximum value. E. Localization of Neur (anti-Neur, green) and Histone2B::RFP expressed under the neur promoter (red) in wild-type SOP daughter cells $(n=10)$ and in strat SOP daughter cells expressing pnrGAL4>AP-4 $7^{d s R N A}(\mathrm{n}=3)$. The same phenotype is observed in the pnr-GAL4>strat ${ }^{d s R N A}, A P$ $47^{\text {dsRNA }}$ (data not shown, $n=9$ ). Yellow arrows point to the enrichment of Neur at the basolateral interface between SOP daughter cells and white arrows point to the enrichment of Neur at the apical interface between SOP daughter cells. Dashed white lines highlight SOP and SOP daughter cells. Scale bar is $5 \mu \mathrm{m}$.

Figure 5: Photoconverted apical Notch is detected in SOP daughter cells nuclei in absence of Strat and AP-1

A-A'. Schematic representations and time-lapse imaging of wild-type and pnrGAL4>strat $t^{d s R N A}, A P-47^{d s R N A}$ SOP daughter cells expressing GAP43::IR. Green and red rectangles represent the photoconversion area. B. Photoconversion at $t_{0}+30$ minutes of SOP daughter cells nuclei in wild-type and pnr-GAL4>strat ${ }^{d s R N A}, A P-47^{d s R N A}$. Dashed yellow rectangles represent the photoconverted $\mathrm{ROI}$, dashed white circles represent the area where nuclei signal has been measured and dashed white lines highlight SOP daughter cells. The red signal of NimMaple3 is measured before and after photoconversion. B'. Plot of photoconverted nuclear signal in pllb and plla cells in wild-type ( $\mathrm{n}=13,3$ pupae) and pnrGAL4>strat ${ }^{d s R N A}, A P-47^{d s R N A}$ ( $n=12,4$ pupae). C-C'”. Photoconversion of apical NimMaple3 and nuclei signal measurement in pnr-GAL4>strat ${ }^{d s R N A}, A P-47^{d s R N A}$ SOP daughter cells. C. Schematic representation of apical photoconversion assays in pnr-GAL4>strat ${ }^{d s R N A}, A P$ $47^{d s R N A}$ SOP daughter cells. Apical photoconversions were performed at 15, 20, 25 and 30 minutes after anaphase transition. Before photoconversion, z-stacks were performed to localize the apical interface using GAP43::IR. A z-stack was then acquired 35 minutes after the anaphase onset to quantify nuclear NimMaple3. C'. Time-lapse imaging of pnrGAL4>strat ${ }^{d s R N A}$, AP-4 $7^{d s R N A}$ SOPs cells expressing GAP43::IR before apical photoconversions. Yellow square highlights the SOP where apical Notch is photoconverted and the blue square highlights the SOP where Notch is not photoconverted and serves as reference. C'. Photoconversion of apical NimMaple3 at $t_{0}+15,20,25$ and 30 minutes in pnr-GAL4>strat ${ }^{d s R N A}, A P-47^{d s R N A}$ SOPs cells expressing GAP43::IR ( $\mathrm{n}=14,4$ pupae). White arrows show Notch present at the apical plla-pllb interface. C'”. Measurements of nuclei signal (nuclei are delimited by dashed white circles) in photoconverted cell (upper panel, yellow) and non-photoconverted cell (lower panel, blue). pllb or plla-like are localized at the left of the apical interface and plla are localized at the right of the apical interface. $\mathbf{D}$. 
plla/pllb ratio values of photoconverted nuclear NimMaple3 at $t_{0}+30$ minutes upon apical NimMaple3 photoconversion in pnr-GAL4>strat ${ }^{d s R N A}, A P-47^{d s R N A}(\mathrm{n}=14,4$ pupae). D'. Plot of photoconverted nuclear signal in pllb/plla-like and plla cells in pnr-GAL4>strat ${ }^{d s R N A}, A P$ $47^{d s R N A}$ ( $n=14,4$ pupae). Scale bar is $5 \mu \mathrm{m}$. $t_{0}$ represents the SOP anaphase onset. In $\mathbf{B}^{\prime}$ and D-D', data are represented as mean $+/$ - SD. (ns $\geq 0.05$ and ${ }^{* *} P<0.01$ ). Because GAP43::IR is excluded from the nuclei, nuclei were defined using an area within cells where the GAP43::IR signal background is the lowest.

\section{Figure 6: Model for the roles of Strat and AP-1 in the transport of Notch signaling components}

Schematic representation of Strat and AP-1 function in the transport of Notch, Delta and Spdo in wild-type and in absence of Strat and AP-1. Nuclei of cells are represented with a blue circle (Notch positive) or in red (Notch negative). AP-2, which is asymmetrically enriched in pllb (Berdnik et al., 2002), promotes the endocytosis of Notch/Spdo (black arrows), while AP-1 regulates their recycling to basolateral membrane (green arrows), a step negatively regulated by Numb in pllb cells. The traffic of Notch, Delta and Spdo that is dependent on Strat and AP-1 is represented in orange or in green arrows, respectively. In absence of Strat and AP-1, the mistrafficking of Notch, Delta and Spdo is represented by pink arrows. Notch and Spdo are also found in apical dotted compartment, and their possible routing to and from there is depicted by pink dashed arrow (TGN: for the trans-Golgi network and $\mathrm{RE}$ : recycling endosomes).

\section{References}

Ang, A.L., Folsch, H., Koivisto, U.M., Pypaert, M., Mellman, I., 2003. The Rab8 GTPase selectively regulates AP-1B-dependent basolateral transport in polarized Madin-Darby canine kidney cells. The Journal of cell biology 163, 339-350.

Artavanis-Tsakonas, S., Rand, M.D., Lake, R.J., 1999. Notch signaling: cell fate control and signal integration in development. Science 284, 770-776.

Bellaiche, Y., Radovic, A., Woods, D.F., Hough, C.D., Parmentier, M.L., O'Kane, C.J., Bryant, P.J., Schweisguth, F., 2001. The Partner of Inscuteable/Discs-large complex is required to establish planar polarity during asymmetric cell division in Drosophila. Cell 106, 355-366.

Bellec, K., Gicquel, I., Le Borgne, R., 2018. Stratum recruits Rab8 at Golgi exit sites to regulate the basolateral sorting of Notch and Sanpodo. Development 145.

Benhra, N., Lallet, L., Cotton, M., Le Bras, S., Dussert, A., Le Borgne, R., 2011. AP-1 controls the trafficking of Notch and Sanpodo toward E-Cadherin junctions in sensory organ precursors. Current biology : CB 21, 1-9. 
Berdnik, D., Torok, T., Gonzalez-Gaitan, M., Knoblich, J.A., 2002. The endocytic protein alpha-Adaptin is required for numb-mediated asymmetric cell division in Drosophila. Developmental cell 3, 221-231.

Besson, C., Bernard, F., Corson, F., Rouault, H., Reynaud, E., Keder, A., Mazouni, K., Schweisguth, F., 2015. Planar Cell Polarity Breaks the Symmetry of PAR Protein Distribution prior to Mitosis in Drosophila Sensory Organ Precursor Cells. Current biology : CB 25, 1104-1110.

Bray, S., 1998. Notch signalling in Drosophila: three ways to use a pathway. Seminars in cell \& developmental biology 9, 591-597.

Burgess, J., Jauregui, M., Tan, J., Rollins, J., Lallet, S., Leventis, P.A., Boulianne, G.L., Chang, H.C., Le Borgne, R., Krämer, H., Brill, J.A., 2011. AP-1 and clathrin are essential for secretory granule biogenesis in Drosophila. Mol Biol Cell. 22, 2094-2105.

Carvajal-Gonzalez, J.M., Gravotta, D., Mattera, R., Diaz, F., Perez Bay, A., Roman, A.C., Schreiner, R.P., Thuenauer, R., Bonifacino, J.S., Rodriguez-Boulan, E., 2012. Basolateral sorting of the coxsackie and adenovirus receptor through interaction of a canonical YXXPhi motif with the clathrin adaptors AP-1A and AP-1B. Proceedings of the National Academy of Sciences of the United States of America 109, 3820-3825.

Castillon, G.A., Burriat-Couleru, P., Abegg, D., Criado Santos, N., Watanabe, R., 2018. Clathrin and AP1 are required for apical sorting of glycosyl phosphatidyl inositolanchored proteins in biosynthetic and recycling routes in Madin-Darby canine kidney cells. Traffic 19, 215-228.

Cotton, M., Benhra, N., Le Borgne, R., 2013. Numb inhibits the recycling of Sanpodo in Drosophila sensory organ precursor. Current biology : CB 23, 581-587.

Couturier, L., Mazouni, K., Schweisguth, F., 2013. Numb localizes at endosomes and controls the endosomal sorting of notch after asymmetric division in Drosophila. Current biology : CB 23, 588-593.

Couturier, L., Vodovar, N., Schweisguth, F., 2012. Endocytosis by Numb breaks Notch symmetry at cytokinesis. Nature cell biology 14, 131-139.

Devergne, O., Sun, G.H., Schupbach, T., 2017. Stratum, a Homolog of the Human GEF Mss4, Partnered with Rab8, Controls the Basal Restriction of Basement Membrane Proteins in Epithelial Cells. Cell reports 18, 1831-1839.

Gillard, G., Shafaq-Zadah, M., Nicolle, O., Damaj, R., Pecreaux, J., Michaux, G., 2015. Control of E-cadherin apical localisation and morphogenesis by a SOAP-1/AP-1/clathrin pathway in C. elegans epidermal cells. Development 142, 1684-1694.

Gordon, W.R., Zimmerman, B., He, L., Miles, L.J., Huang, J., Tiyanont, K., McArthur, D.G., Aster, J.C., Perrimon, N., Loparo, J.J., Blacklow, S.C., 2015. Mechanical Allostery: Evidence for a Force Requirement in the Proteolytic Activation of Notch. Developmental cell 33, 729-736.

Gratz, S.J., Cummings, A.M., Nguyen, J.N., Hamm, D.C., Donohue, L.K., Harrison, M.M., Wildonger, J., O'Connor-Giles, K.M., 2013a. Genome engineering of Drosophila with the CRISPR RNA-guided Cas9 nuclease. Genetics 194, 1029-1035.

Gratz, S.J., Wildonger, J., Harrison, M.M., O'Connor-Giles, K.M., 2013b. CRISPR/Cas9mediated genome engineering and the promise of designer flies on demand. Fly 7, 249255.

Gupta-Rossi, N., Le Bail, O., Gonen, H., Brou, C., Logeat, F., Six, E., Ciechanover, A., Israel, A., 2001. Functional interaction between SEL-10, an F-box protein, and the nuclear form of activated Notch1 receptor. The Journal of biological chemistry 276, 34371-34378.

Henry, L., Sheff, D.R., 2008. Rab8 regulates basolateral secretory, but not recycling, traffic at the recycling endosome. Molecular biology of the cell 19, 2059-2068. 
Holloway, Z.G., Velayos-Baeza, A., Howell, G.J., Levecque, C., Ponnambalam, S., Sztul, E., Monaco, A.P., 2013. Trafficking of the Menkes copper transporter ATP7A is regulated by clathrin-, AP-2-, AP-1-, and Rab22-dependent steps. Molecular biology of the cell 24, 1735-1748, S1731-1738.

Huber, L.A., de Hoop, M.J., Dupree, P., Zerial, M., Simons, K., Dotti, C., 1993. Protein transport to the dendritic plasma membrane of cultured neurons is regulated by rab8p. The Journal of cell biology 123, 47-55.

Hutterer, A., Knoblich, J.A., 2005. Numb and alpha-Adaptin regulate Sanpodo endocytosis to specify cell fate in Drosophila external sensory organs. EMBO reports 6, 836-842.

Jafar-Nejad, H., Andrews, H.K., Acar, M., Bayat, V., Wirtz-Peitz, F., Mehta, S.Q., Knoblich, J.A., Bellen, H.J., 2005. Sec15, a component of the exocyst, promotes notch signaling during the asymmetric division of Drosophila sensory organ precursors. Developmental cell 9, 351-363.

Jarriault, S., Brou, C., Logeat, F., Schroeter, E.H., Kopan, R., Israel, A., 1995. Signalling downstream of activated mammalian Notch. Nature 377, 355-358.

Johnson, S.A., Zitserman, D., Roegiers, F., 2016. Numb regulates the balance between Notch recycling and late-endosome targeting in Drosophila neural progenitor cells. Molecular biology of the cell 27, 2857-2866.

Kopan, R., Ilagan, M.X., 2009. The canonical Notch signaling pathway: unfolding the activation mechanism. Cell 137, 216-233.

Lai, E.C., Deblandre, G.A., Kintner, C., Rubin, G.M., 2001. Drosophila neuralized is a ubiquitin ligase that promotes the internalization and degradation of delta. Developmental cell 1, 783-794.

Langevin, J., Le Borgne, R., Rosenfeld, F., Gho, M., Schweisguth, F., Bellaiche, Y., 2005. Lethal giant larvae controls the localization of notch-signaling regulators numb, neuralized, and Sanpodo in Drosophila sensory-organ precursor cells. Current biology : CB 15, 955-962.

Langridge, P.D., Struhl, G., 2017. Epsin-Dependent Ligand Endocytosis Activates Notch by Force. Cell 171, 1383-1396 e1312.

Le Borgne, R., Schweisguth, F., 2003. Unequal segregation of Neuralized biases Notch activation during asymmetric cell division. Developmental cell 5, 139-148.

Meloty-Kapella, L., Shergill, B., Kuon, J., Botvinick, E., Weinmaster, G., 2012. Notch ligand endocytosis generates mechanical pulling force dependent on dynamin, epsins, and actin. Developmental cell 22, 1299-1312.

Mumm, J.S., Schroeter, E.H., Saxena, M.T., Griesemer, A., Tian, X., Pan, D.J., Ray, W.J., Kopan, R., 2000. A ligand-induced extracellular cleavage regulates gamma-secretaselike proteolytic activation of Notch1. Molecular cell 5, 197-206.

Nakajo, A., Yoshimura, S., Togawa, H., Kunii, M., Iwano, T., Izumi, A., Noguchi, Y., Watanabe, A., Goto, A., Sato, T., Harada, A., 2016. EHBP1L1 coordinates Rab8 and Bin1 to regulate apical-directed transport in polarized epithelial cells. The Journal of cell biology 212, 297-306.

Norgate, M., Lee, E., Southon, A., Farlow, A., Batterham, P., Camakaris, J., Burke, R., 2006. Essential roles in development and pigmentation for the Drosophila copper transporter DmATP7. Molecular biology of the cell 17, 475-484.

O'Connor-Giles, K.M., Skeath, J.B., 2003. Numb inhibits membrane localization of Sanpodo, a four-pass transmembrane protein, to promote asymmetric divisions in Drosophila. Developmental cell 5, 231-243.

Rajan, A., Tien, A.C., Haueter, C.M., Schulze, K.L., Bellen, H.J., 2009. The Arp2/3 complex and WASp are required for apical trafficking of Delta into microvilli during cell fate specification of sensory organ precursors. Nature cell biology 11, 815-824. 
Rhyu, M.S., Jan, L.Y., Jan, Y.N., 1994. Asymmetric distribution of numb protein during division of the sensory organ precursor cell confers distinct fates to daughter cells [see comments]. Cell 76, 477-491.

Sato, T., Iwano, T., Kunii, M., Matsuda, S., Mizuguchi, R., Jung, Y., Hagiwara, H., Yoshihara, Y., Yuzaki, M., Harada, R., Harada, A., 2014. Rab8a and Rab8b are essential for several apical transport pathways but insufficient for ciliogenesis. Journal of cell science $127,422-431$.

Sato, T., Mushiake, S., Kato, Y., Sato, K., Sato, M., Takeda, N., Ozono, K., Miki, K., Kubo, Y., Tsuji, A., Harada, R., Harada, A., 2007. The Rab8 GTPase regulates apical protein localization in intestinal cells. Nature 448, 366-369.

Schweisguth, F., 2015. Asymmetric cell division in the Drosophila bristle lineage: from the polarization of sensory organ precursor cells to Notch-mediated binary fate decision. Wiley interdisciplinary reviews. Developmental biology 4, 299-309.

Seo, D., Southard, K.M., Kim, J.W., Lee, H.J., Farlow, J., Lee, J.U., Litt, D.B., Haas, T., Alivisatos, A.P., Cheon, J., Gartner, Z.J., Jun, Y.W., 2016. A Mechanogenetic Toolkit for Interrogating Cell Signaling in Space and Time. Cell 165, 1507-1518.

Shergill, B., Meloty-Kapella, L., Musse, A.A., Weinmaster, G., Botvinick, E., 2012. Optical tweezers studies on Notch: single-molecule interaction strength is independent of ligand endocytosis. Developmental cell 22, 1313-1320.

Struhl, G., Adachi, A., 2000. Requirements for presenilin-dependent cleavage of notch and other transmembrane proteins. Molecular cell 6, 625-636.

Trylinski, M., Mazouni, K., Schweisguth, F., 2017. Intra-lineage Fate Decisions Involve Activation of Notch Receptors Basal to the Midbody in Drosophila Sensory Organ Precursor Cells. Current biology : CB 27, 2239-2247 e2233.

Upadhyay, A., Kandachar, V., Zitserman, D., Tong, X., Roegiers, F., 2013. Sanpodo controls sensory organ precursor fate by directing Notch trafficking and binding gammasecretase. The Journal of cell biology 201, 439-448.

Wang, X., Ha, T., 2013. Defining single molecular forces required to activate integrin and notch signaling. Science 340, 991-994.

Zhang, H., Kim, A., Abraham, N., Khan, L.A., Hall, D.H., Fleming, J.T., Gobel, V., 2012. Clathrin and AP-1 regulate apical polarity and lumen formation during C. elegans tubulogenesis. Development 139, 2071-2083. 


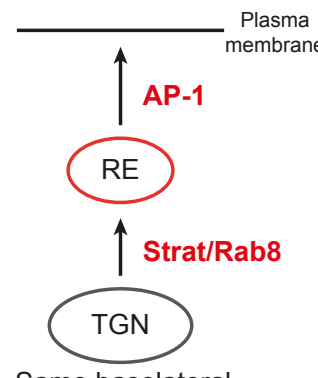

Same basolateral transport pathway

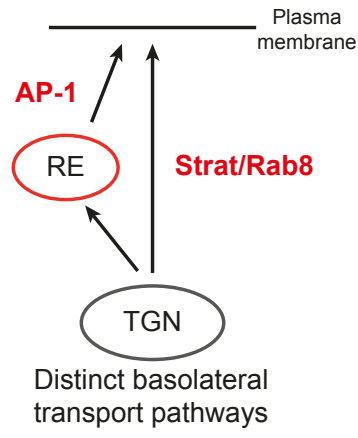

strat

pnr-GAL4 $>A P-47^{d S R N A}$
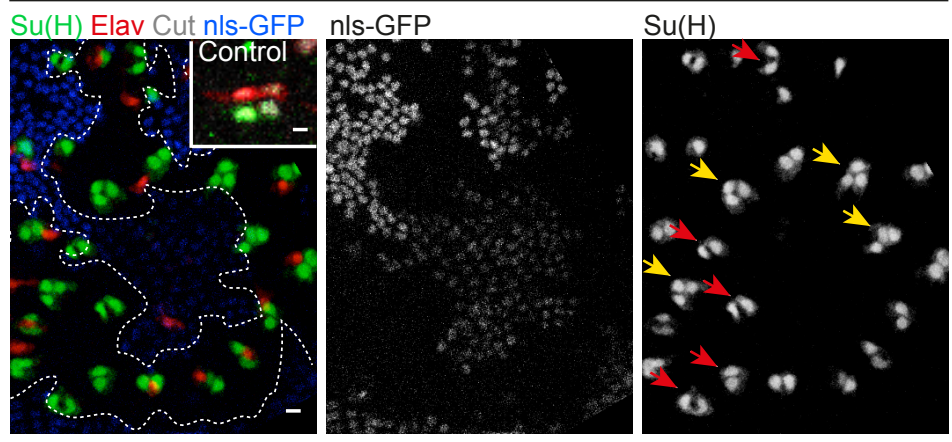

C

\begin{tabular}{|c|c|c|c|c|c|}
\cline { 2 - 6 } \multicolumn{1}{l|}{} & $\begin{array}{c}\text { Control } \\
\mathrm{n}=204\end{array}$ & $\begin{array}{c}\text { strat } \\
\text { pnr-GAL4/+ } \\
\mathrm{n}=328\end{array}$ & $\begin{array}{c}\text { pnr-GAL4> } \\
\text { AP-47dsRNA } \\
\mathrm{n}=362\end{array}$ & $\begin{array}{c}\text { strat, pnr-GAL4> } \\
\text { AP-47dsRNA } \\
\mathrm{n}=254\end{array}$ & $\begin{array}{c}\text { pnr-GAL4>stratsRNA } \\
\text { AP-47dsRNA } \\
\mathrm{n}=144\end{array}$ \\
\hline $0 \mathrm{Su}(\mathrm{H})$ & 0 & 41 & 0 & 0 & 0 \\
\hline $1 \mathrm{Su}(\mathrm{H})$ & 204 & 265 & 335 & 130 & 57 \\
\hline $2 \mathrm{Su}(\mathrm{H})$ & 0 & 22 & 23 & 87 & 47 \\
\hline $3 / 4 \mathrm{Su}(\mathrm{H})$ & 0 & 0 & 4 & 37 & 40 \\
\hline $\begin{array}{c}\% \text { of } \\
\text { Notch GOF }\end{array}$ & $0 \%$ & $6,7 \%$ & $7,5 \%$ & $49 \%$ & $60 \%$ \\
\hline
\end{tabular}




\section{A NiGFP nls-GFP NeurH2B::RFP}

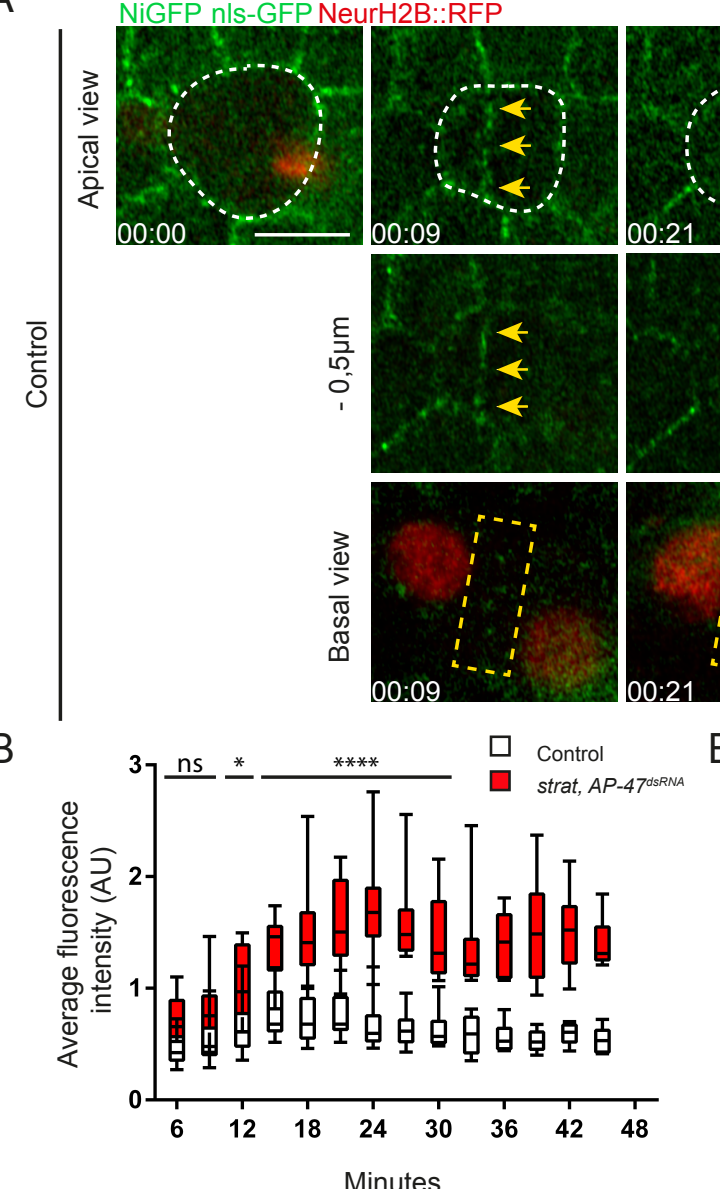

C
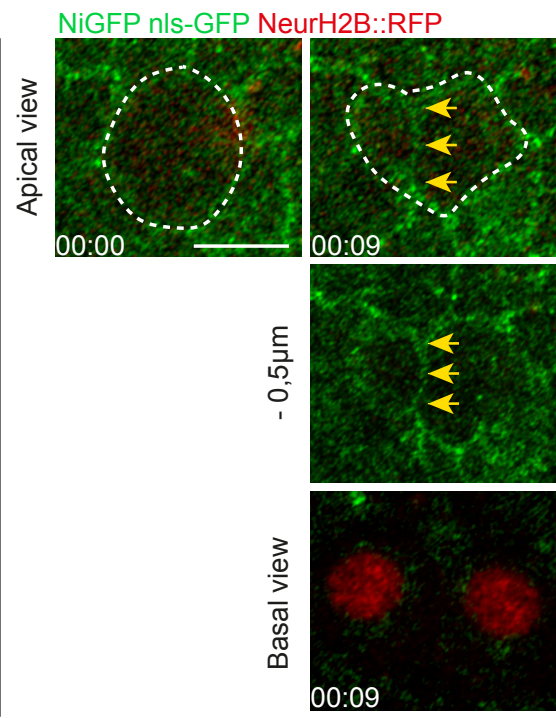
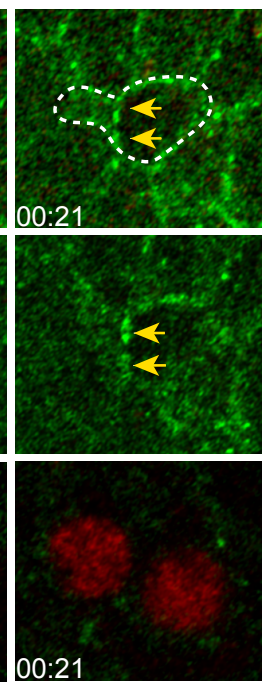
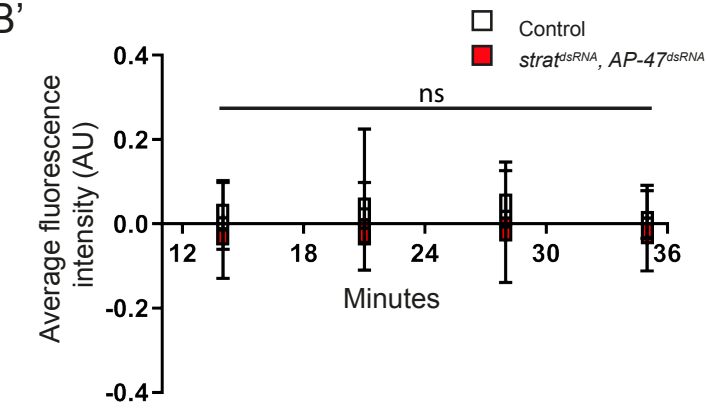

C'

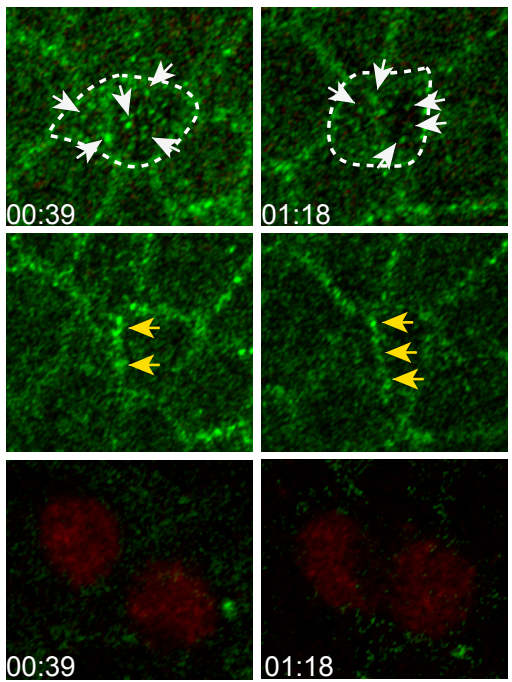

Control

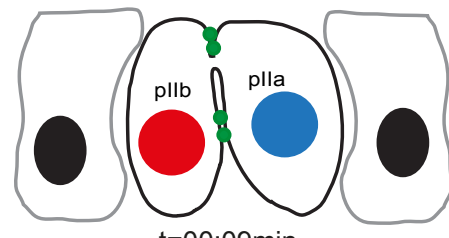

$\mathrm{t}=00: 09 \mathrm{~min}$

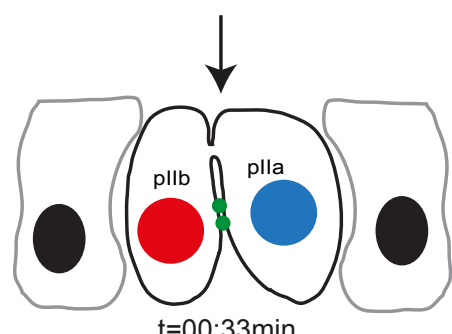

- Notch

\section{pnr-GAL4 $>A P-47^{d s R N A}$}

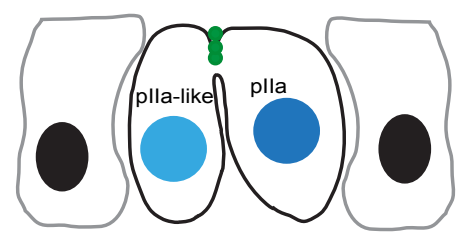

$\mathrm{t}=00: 09 \mathrm{~min}$

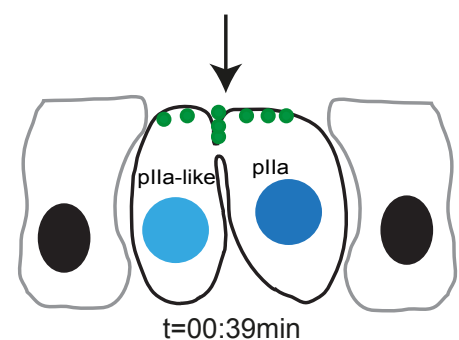

- Notch $\mathrm{t}=00: 39$ min 

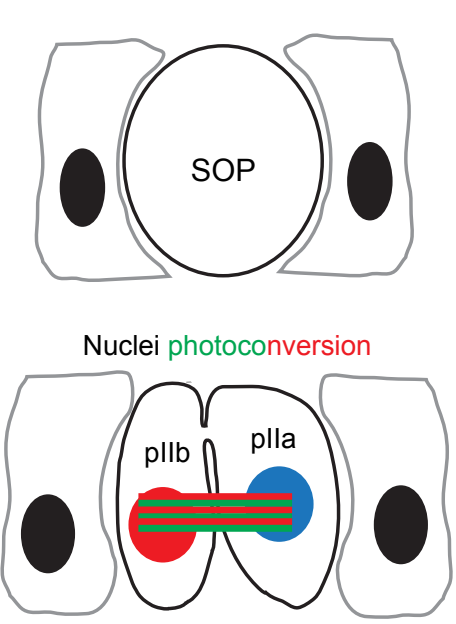
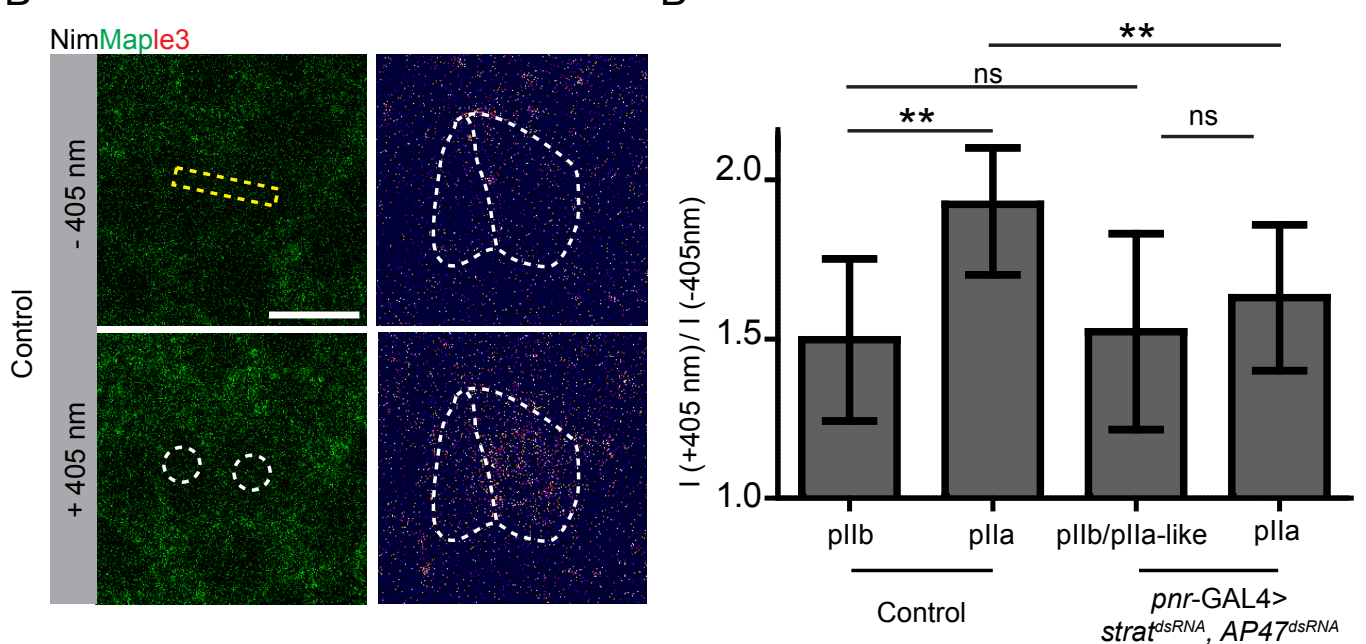

stratdsRNA,$A P 47^{d s R N A}$

$A^{\prime}$

GAP43::IR
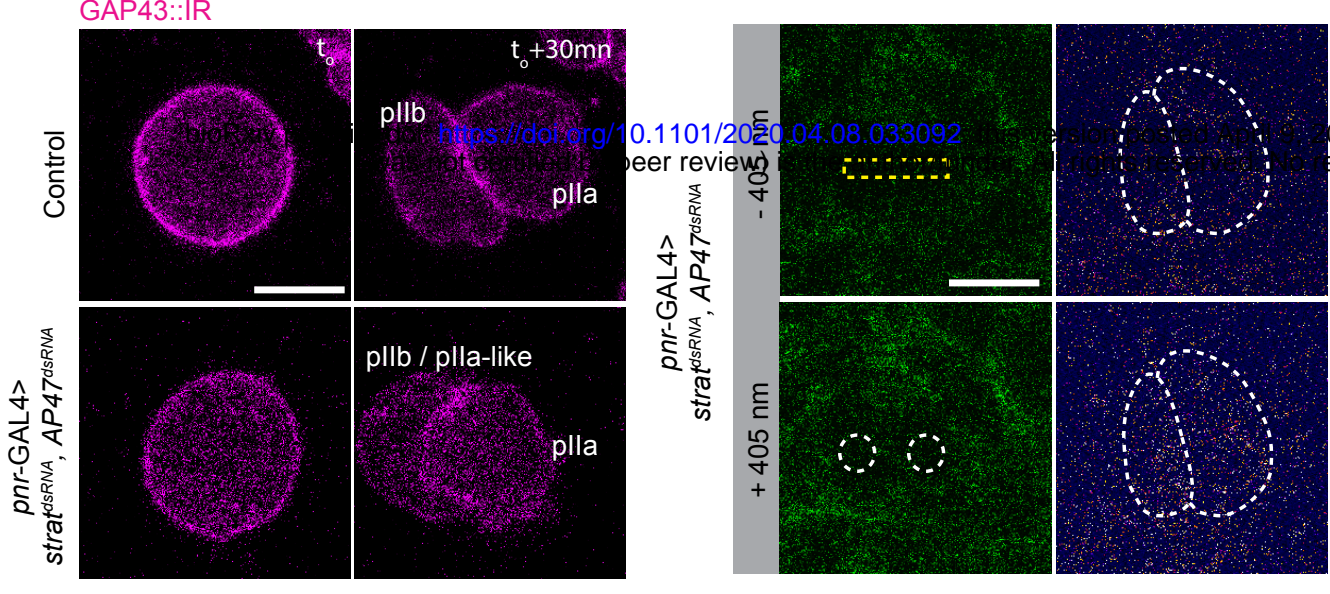

20. The copyright holder for this preprint (which euse allowed without permission.

\section{C}

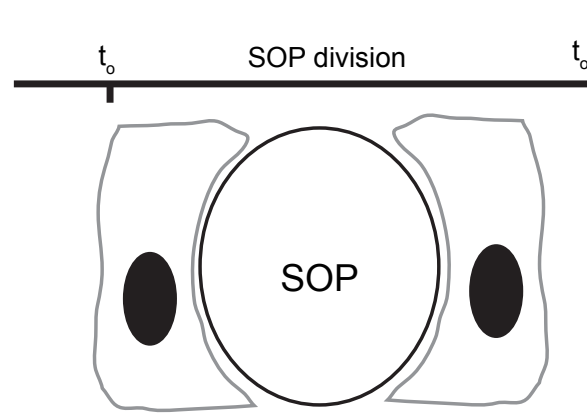

$C^{\prime}$

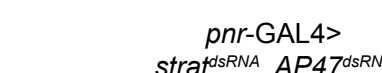

pnr-GAL4>
GAP43::IR strat ${ }^{d s R N A}, A P 47^{d s R N A}$

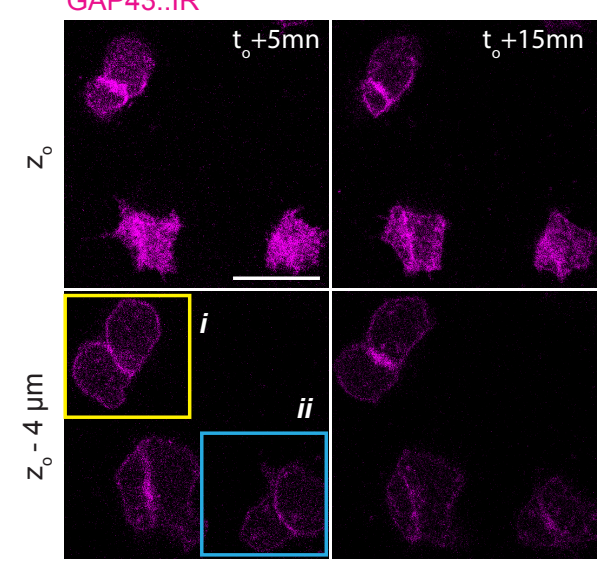

$C^{\prime \prime}$
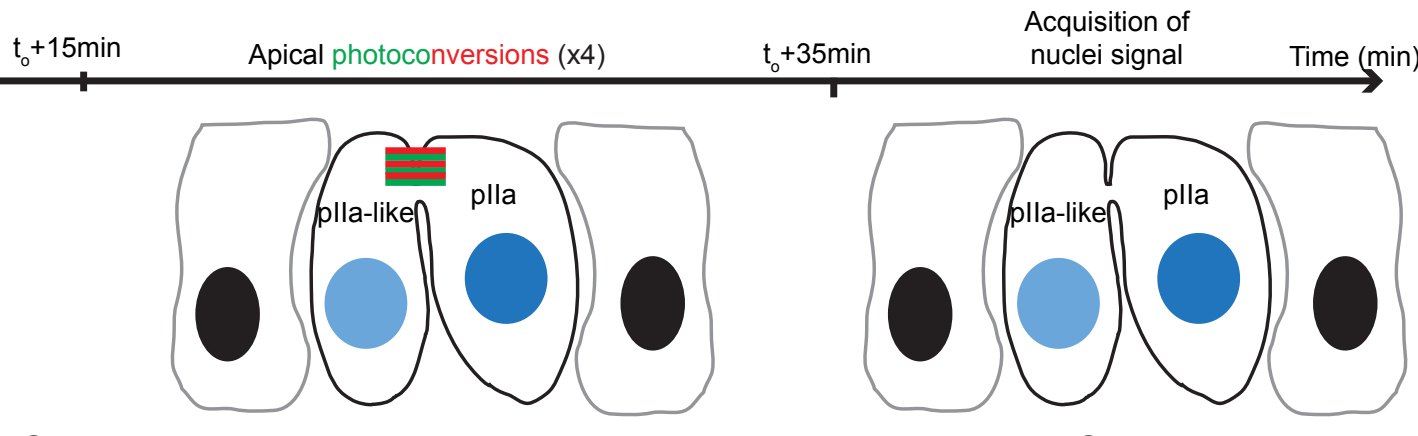

C'”
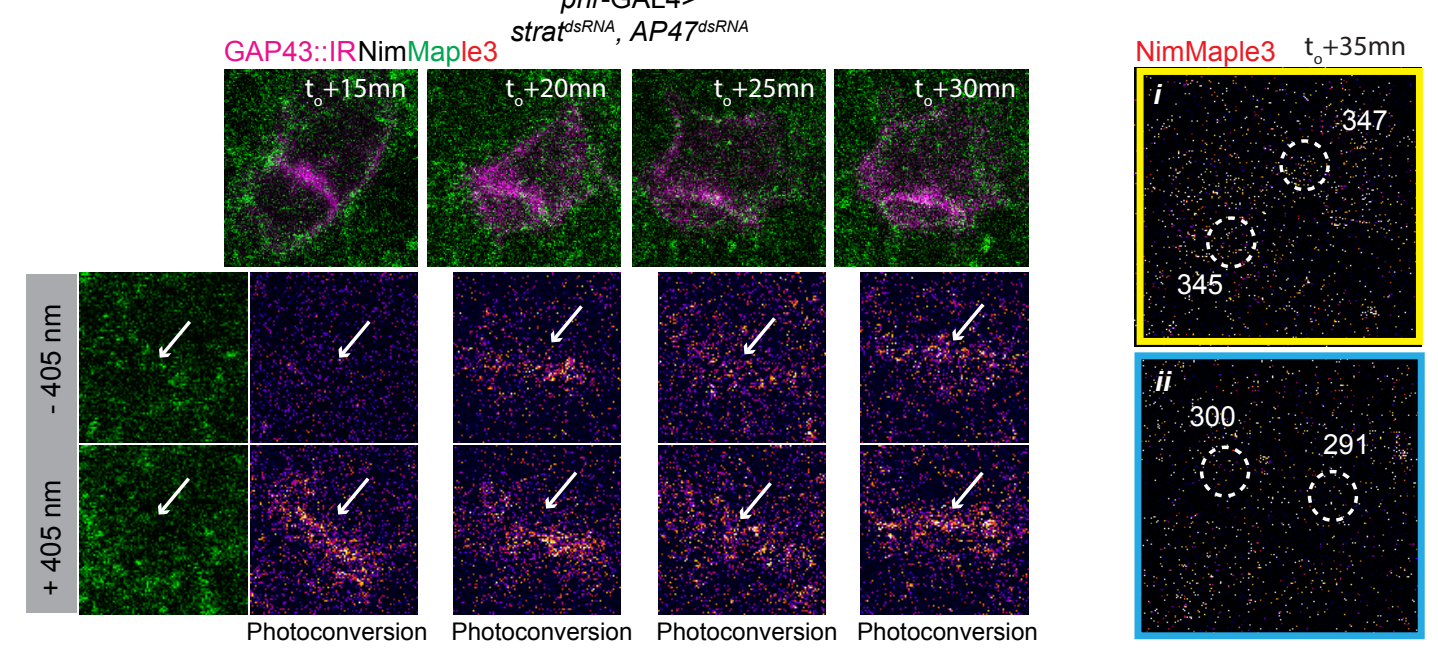

D
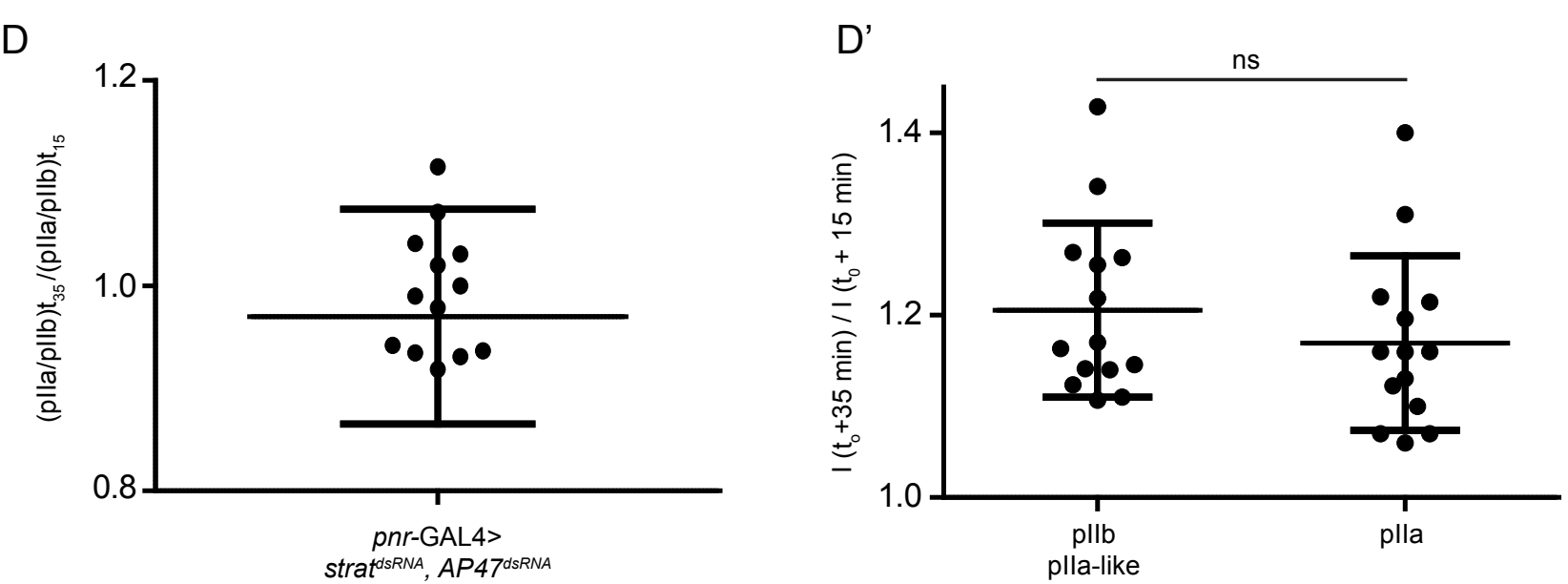
Control

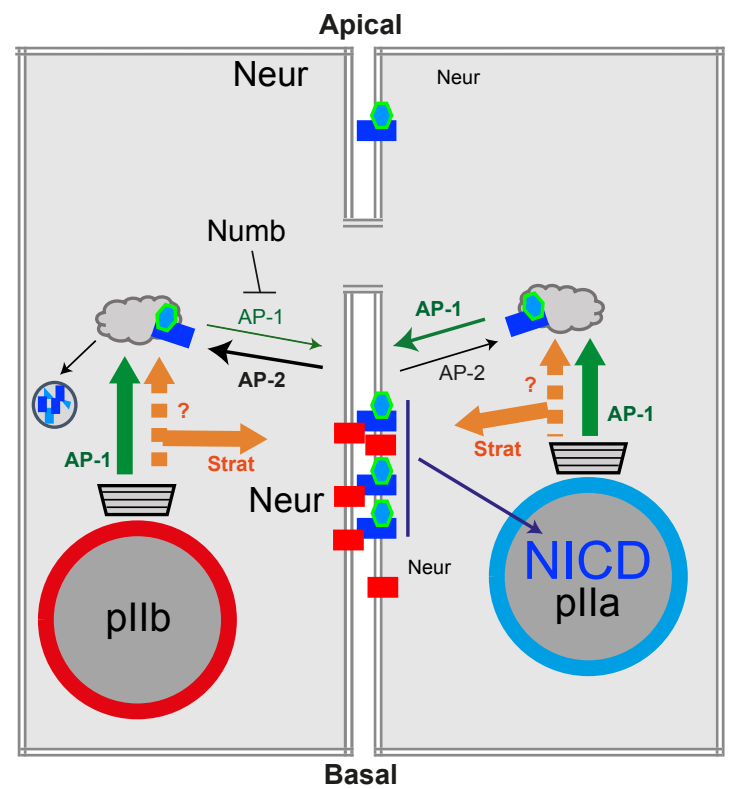

\section{Loss of Stratum and AP-1}

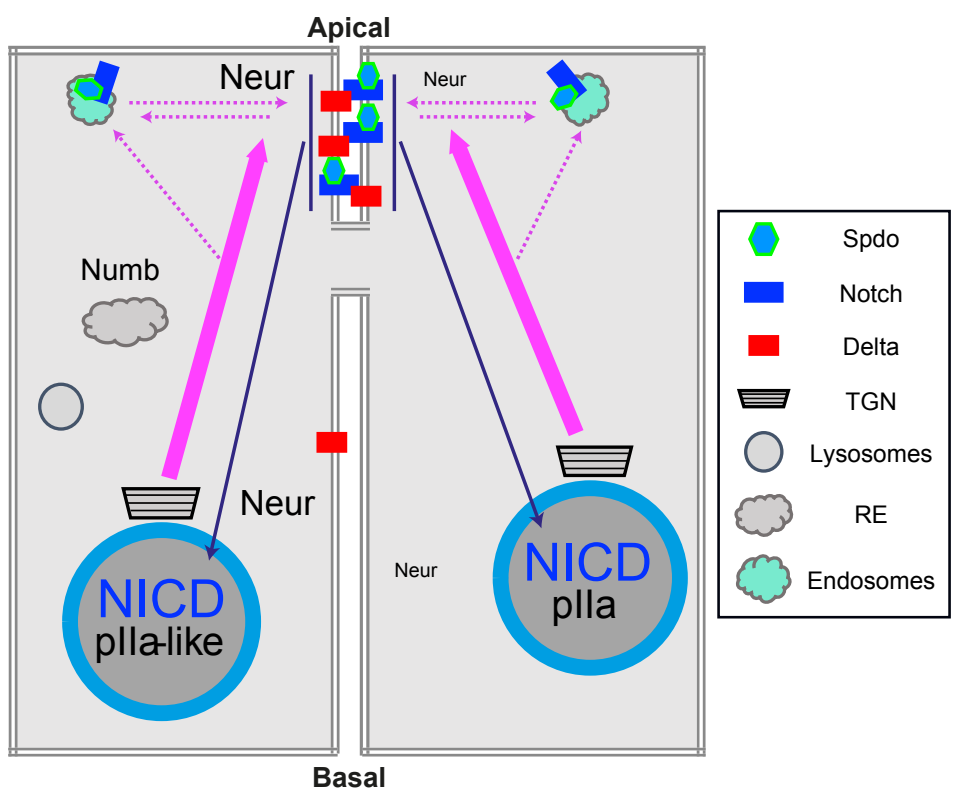

